Errata

Finite Energy Solutions for Interacting Yang-Mills and Dirac Fields on Minkowski Space

K. Meetz

Z. Phys. C - Particles and Fields 6, 41 (1980)

In (40) replace $S O(3)$ by $S U(2)$. Equation (41) should read

$U(n)=n_{4}+i n^{a} \tau^{a}$

( $\tau^{a}$ Pauli matrices). In (65) put

$\omega=\left[\left(1+x^{2}\right)^{2}+4 x^{2}\right]^{-1 / 2}$.

A factor $\omega^{2}$ has been omitted in the second line of (65). In (75)

replace $i$ by 1 .

\title{
Superalgebras of Non-Abelian Gauge Theories in the Manifestly-Covariant Canonical Formalism
}

N. Nakanishi, I. Ojima

Z. Phys. C - Particles and Fields 6. 155 (1980)

On p. 158 , in line next to (5.2), delete $t_{b}^{a} \equiv \eta^{a c} t_{c b}$.

On p. 159 , in (5.27), $t_{a}^{b}$ should read $t_{a}^{b}$.

On p. 159 , in (5.31), $\bar{t}_{a}^{b}$ should be read $\bar{t}_{a}^{b}$

On p. 159, in (5.33) and in (5.34), $\bar{t}_{a}^{c}$ and $\bar{t}_{b}^{c}$ should be read $\bar{t}_{a}^{c}$ and $\bar{t}_{b}^{c}$, respectively. 